\title{
LOCAL REGULARITY OF SOLUTIONS TO TIME-DEPENDENT SCHRÖDINGER EQUATIONS WITH SMOOTH POTENTIALS
}

\author{
Peter Sjögren and Per Sjölin
}

\begin{abstract}
Consider solutions to the Schrödinger equation $i \partial u / \partial t=-P u+V u$ in a half-space $\left\{(x, t) \in \mathbf{R}^{n} \times \mathbf{R}_{+}\right\}$with given boundary values $u=f$ on $\mathbf{R}^{n}$. Here $P$ is an elliptic constant-coefficient operator in $x$, and $V=V(x)$ is a suitable potential. We prove several results about local regularity and boundary behaviour which were known in the case $V=0$. In particular, if $f$ belongs to a Sobolev space, then $u$ is locally in a mixed Sobolev space. Moreover, $u$ converges to its boundary values along quasi-every vertical ray, and the corresponding maximal function can be estimated.
\end{abstract}

\section{Introduction}

We define the Fourier transform in $\mathbf{R}^{n}$ by setting

$$
\hat{f}(\xi)=\int_{\mathbf{R}^{n}} e^{-i \xi \cdot x} f(x) d x
$$

and also introduce Sobolev spaces $H_{s}=H_{s}\left(\mathbf{R}^{n}\right), s \in \mathbf{R}$, by defining the norm

$$
\|f\|_{H_{s}}=\left(\int_{\mathbf{R}^{n}}\left(1+|\xi|^{2}\right)^{s}|\hat{f}(\xi)|^{2} d \xi\right)^{1 / 2} .
$$

Let $p$ be a polynomial in $\mathbf{R}^{n}$ which is real and elliptic, i.e. its principal part does not vanish in $\mathbf{R}^{n} \backslash\{0\}$. We assume that the degree $m$ of $p$ is at least 2 . Then set $P=p(D)$, where $D=\left(D_{1}, \ldots, D_{n}\right)$ and $D_{k}=-i \partial / \partial x_{k}$.

Let $V$ be a real-valued function in $C^{\infty}\left(\mathbf{R}^{n}\right)$ with $D^{\alpha} V$ bounded for every $\alpha$. We define an operator $H=-P+V$ by setting $H f=-P f+V f$ for $f \in H_{m}$.

Then $H$ is a self-adjoint operator on $L^{2}\left(\mathbf{R}^{n}\right)$ and $e^{-i t H}$ is a unitary operator for $t \in \mathbf{R}$. We set $u(\cdot, t)=e^{-i t H} f, f \in L^{2}\left(\mathbf{R}^{n}\right)$. Then $u$ is a measurable function in $\mathbf{R}^{n+1}$, and it is well known that $u$ satifies the Schrödinger equation $i \partial u / \partial t=H u$ for $f \in H_{m}$, where the derivative is taken in the $L^{2}$ sense. Taking the derivatives in the distribution sense, one also has $i \partial u / \partial t=-P u+V u$ for $f \in L^{2}$.

AMS classification numbers: $35 \mathrm{~K} 30,42 \mathrm{~B} 25$.

The first author is supported by Naturvetenskapliga Forskningsrådet. 
We shall here study the regularity of $u$ when the initial value $f$ belongs to Sobolev spaces $H_{s}$. Also the pointwise convergence of $u(\cdot, t)$ to $f$ as $t \rightarrow 0$ will be discussed.

To formulate the results, we introduce mixed Sobolev spaces $H_{\varrho, r}$ for $\varrho \geq 0$, $r \geq 0$. We set $H_{\ell, r}=H_{\varrho, r}\left(\mathbf{R}^{n} \times \mathbf{R}\right)=\left(G_{\varrho} \otimes G_{r}\right) * L^{2}\left(\mathbf{R}^{n+1}\right)$, where $G_{\varrho}$ and $G_{r}$ are Bessel kernels in $\mathbf{R}^{n}$ and $\mathbf{R}$, respectively. The Bessel kernel is given by the formula $\hat{G}_{s}(\xi)=\left(1+|\xi|^{2}\right)^{-s / 2}$, and the norm in $H_{Q, r}$ is the obvious one.

We introduce the class

$$
\begin{aligned}
& \mathcal{A}=\left\{\varphi \in C^{\infty}\left(\mathbf{R}^{n}\right) ; \text { there exists } \varepsilon>0\right. \text { such that } \\
&\left.\left|D^{\alpha} \varphi(x)\right| \leq C_{\alpha}(1+|x|)^{-1 / 2-\varepsilon} \text { for every } \alpha\right\}
\end{aligned}
$$

and set

$$
S f(x, t)=\varphi(x) \psi(t) u(x, t),
$$

where we assume that $\varphi \in \mathcal{A}$ and $\psi \in C_{0}^{\infty}(\mathbf{R})$. Let $\mathcal{S}$ denote the Schwartz class.

We then have the following result.

Theorem 1. If $\varrho \geq 0, r \geq 0$, then

$$
\|S f\|_{H_{\ell, r}} \leq C\|f\|_{H_{Q+m r-(m-1) / 2}}, \quad f \in \mathcal{S} .
$$

Here the constant $C$ depends on $\varphi$ and $\psi$.

Theorem 1 expresses a local smoothing property for the Schrödinger equation. For instance, taking $\varrho=r=0$ in (1) we obtain

$$
\|S f\|_{L^{2}\left(\mathbf{R}^{n+1}\right)} \leq C\|f\|_{H_{-(m-1) / 2}}
$$

and $\varrho=(m-1) / 2, r=0$ yields

$$
\|S f\|_{H_{(m-1) / 2,0}} \leq C\|f\|_{L^{2}\left(\mathbf{R}^{n}\right)} .
$$

From (3) it then follows that $\varphi u(\cdot, t) \in H_{(m-1) / 2}\left(\mathbf{R}^{n}\right)$ for almost every $t$ if $f \in$ $L^{2}\left(\mathbf{R}^{n}\right)$.

Setting

$$
u^{*}(x)=\operatorname{ess} \sup _{0<t<1}|u(x, t)|, \quad x \in \mathbf{R}^{n}, \quad f \in \mathcal{S},
$$

we have the following maximal inequality: 
Theorem 2. If $s>\frac{1}{2}$, then for any ball $B \subset \mathbf{R}^{n}$

$$
\left(\int_{B} u^{*}(x)^{2} d x\right)^{1 / 2} \leq C_{B}\|f\|_{H_{\bullet}}, \quad f \in \mathcal{S} .
$$

To formulate our next result, we shall introduce capacities $C_{s}$ for $s>0$. We set

$$
C_{s}(E)=\inf \left\{\|g\|_{2}^{2} ; 0 \leq g \in L^{2}\left(\mathbf{R}^{n}\right), G_{s} * g \geq 1 \text { on } E\right\}, \quad E \subset \mathbf{R}^{n} .
$$

By $C_{s}$-q.e. we mean everywhere except on a set of $C_{s}$-capacity 0 , and similarly for $C_{s}$-q.a.

A function $f \in H_{s}$ can be written as a convolution $f=G_{s} * g$ with $g \in L^{2}$. At $C_{s}$-q.a. points $x$, this convolution is well-defined in the sense that $G_{s} *|g|(x)<\infty$. These well-defined values of $f$ can be recovered if one knows $f$ almost everywhere. In fact, the means of $f$ in small balls centered at $x$ converge to $G_{s} * g(x)$ if $G_{s} *|g|(x)<\infty$.

We shall now describe how to make the solution $u$ to the Schrödinger equation precise by defining it at sufficiently many points. Let $f \in L^{2}\left(\mathbf{R}^{n}\right)$. The function $u$ is measurable and defined a.e. in $\mathbf{R}^{n+1}$. Let $B_{x, t}(\delta)$ be the ball in $\mathbf{R}^{n+1}$ with center $(x, t)$ and radius $\delta$ and let $B_{x, t}^{\prime}(\delta)=\left\{\left(x^{\prime}, t\right) ;\left|x^{\prime}-x\right|<\delta\right\}$ be a horizontal disc. We define the value $u(x, t)$ as the limit as $\delta \rightarrow 0$ of the mean value of $u$ over either $B_{x, t}(\delta)$ or $B_{x, t}^{\prime}(\delta)$, at all points $(x, t)$ where this limit exists. We speak of the ball and the disc method.

Theorem 3. Let $s>\frac{1}{2}$ and take $f \in H_{s}$. Define $u$ as above and make $u$ precise by the ball or the disc method. If $0<\varrho<s-\frac{1}{2}$, then the following holds for $C_{e}$-q.a. $x$ : The function $u$ is defined at every point of the vertical line $\{x\} \times \mathbf{R}$, its restriction to this line is continuous, and its value at $(x, 0)$ is $f(x)$.

We shall also prove that the local smoothing inequality (2) is best possible in the following sense.

Theorem 4. Assume $P=\Delta$ and $V=0$. Define $S f$ as above and assume that

$$
\|S f\|_{L^{2}\left(\mathbf{R}^{n+1}\right)} \leq C\|f\|_{H_{\bullet}}, \quad f \in \mathcal{S},
$$

for all $\varphi \in C_{0}^{\infty}\left(\mathbf{R}^{n}\right)$ and $\psi \in C_{0}^{\infty}(\mathbf{R})$. Then $s \geq-\frac{1}{2}$.

In the cases $n=1$ and $n=2$, Theorem 2 can be improved in the following way.

Theorem 5. Assume $n=1$ or $n=2$. Let $P=\Delta^{k}$, where $k=1,2,3, \ldots$, and define $u^{*}$ as in Theorem 2. Then for any ball $B \subset \mathbf{R}^{n}$

$$
\left(\int_{B} u^{*}(x)^{2} d x\right)^{1 / 2} \leq C_{B}\|f\|_{H_{n / 4}}, \quad f \in \mathcal{S} .
$$


In the case $V=0$ (and $\varphi \in C_{0}^{\infty}$ in Theorem 1) Theorems 1-3 were proved in P. Sjögren and P. Sjölin [5] and Theorem 5 in P. Sjölin [7].

We remark that local smoothness for solutions to Schrödinger equations has also been studied by P. Constantin and J.C. Saut [1], [2].

We also remark that an important tool in the passage from the case $V=0$ to the case of general $V$ is Duhamel's formula for solutions to Schrödinger equations

$$
u(\cdot, t)=e^{i t P} f+\int_{0}^{t} e^{i(t-\tau) P}(-i V u(\cdot, \tau)) d \tau, \quad f \in H_{m}
$$

(cf. [1], [2]).

In [6] we proved Theorem 2 in the case when $n \geq 3$ is odd, $P=\Delta$ and $V \in C_{0}^{\infty}$ is small, with a different method. The proof in [6] was based on results of A. Melin [3], [4] on intertwining operators. Set $H=-\Delta+V$ and $H_{0}=-\Delta$. Melin constructed a bounded linear operator $A$ on $L^{2}$ such that $H A=A H_{0}$. One has

$$
e^{-i t H}=A e^{-i t H_{0}} A^{-1},
$$

and a combination of properties of $A$ and results in [5], [7] for $e^{-i t H_{0}}$ gave Theorem 2 in this special case.

\section{Proof of Theorem 1}

We set

$$
S_{0} f(x, t)=\varphi(x) \psi(t) e^{i t P} f(x), \quad x \in \mathbf{R}^{n}, t \in \mathbf{R}, f \in \mathcal{S}\left(\mathbf{R}^{n}\right),
$$

where $\varphi \in \mathcal{A}$ and $\psi \in C_{0}^{\infty}(\mathbf{R})$. With $\alpha=\frac{1}{2}(m-1)$ one then has

$$
\left\|S_{0} f\right\|_{H_{e, r}} \leq C\|f\|_{H_{e+m r-\alpha}}, \quad \varrho \geq 0, r \geq 0 .
$$

In the case $\varphi \in C_{0}^{\infty}(\mathbf{R})$, (5) was proved in [5]. It is also easily seen that the argument in [5] gives (5) with $\varrho=r=0$ in the case $\varphi=\hat{G}_{s}$ with $s>\frac{1}{2}$. It is then clear that (5) with $\varrho=r=0$ holds for all $\varphi \in \mathcal{A}$, since $|\varphi(x)| \leq C \hat{G}_{s}(x)$ for some $s>\frac{1}{2}$. This result can now be extended as in [5] and gives (5) for all $\varrho \geq 0$, $r \geq 0$.

The conditions on $V$ imply that $V$ is a multiplier on $H_{s}$, i.e.

$$
\|V f\|_{H_{\mathbf{s}}} \leq C_{\boldsymbol{s}}\|f\|_{H_{\mathrm{o}}}, \quad s \in \mathbf{R} .
$$

Next we prove that

$$
\left\|e^{-i t H} f\right\|_{H_{\boldsymbol{s}}} \leq C_{\boldsymbol{s}}\|f\|_{H_{\mathbf{o}}}, \quad t \in \mathbf{R}, s \in \mathbf{R} .
$$


To begin with, it follows from the ellipticity of $P$ that $g \in H_{m}$ if and only if $g \in L^{2}$ and $(-P+V) g \in L^{2}$. More generally,

$$
g \in H_{j m} \text { if and only if } g,(-P+V) g, \ldots,(-P+V)^{j} g \in L^{2},
$$

with a corresponding norm equivalence. Here $j=0,1,2, \ldots$ The "only if" part in (8) follows directly, and we shall prove the "if" part by induction.

Assume that $g,(-P+V) g, \ldots,(-P+V)^{j} \dot{g} \in L^{2}$. Using an induction assumption, we then conclude that $g$ and $(-P+V) g \in H_{(j-1) m}$. It follows that $P g \in H_{(j-1) m}$ and $g \in H_{j m}$. Hence (8) is proved.

For $f \in H_{j m}$ we have

$$
(-P+V)^{k} e^{-i t H} f=e^{-i t H}(-P+V)^{k} f \in L^{2}, \quad 0 \leq k \leq j,
$$

since $(-P+V)^{k} f \in L^{2}$ and $e^{-i t H}$ is unitary on $L^{2}$. It follows that

$$
\left\|e^{-i t H} f\right\|_{H_{j m}} \leq C\|f\|_{H_{j m}}, \quad j=0,1,2, \ldots
$$

and interpolation and duality now give (7).

We shall also verify that the mapping $t \mapsto u(\cdot, t)=e^{-i t H} f$ is continuous from $\mathbf{R}$ to $H_{s}$ if $f \in H_{s}$. Because of (7), it is sufficient to assume that $f \in \mathcal{S}$. We set $v_{t}=u(\cdot, t)-u\left(\cdot, t_{0}\right)$ and may assume that $s>0$. One has

$$
\left\|v_{t}\right\|_{H_{s+1}} \leq C, \quad t \in \mathbf{R}
$$

and

$$
\left\|v_{t}\right\|_{2} \rightarrow 0, \quad t \rightarrow t_{0}
$$

We also have

$$
\begin{aligned}
\left\|v_{t}\right\|_{H_{\bullet}}^{2} & =\int\left(1+|\xi|^{2}\right)^{s}\left|\hat{v}_{t}(\xi)\right|^{2} d \xi \\
& \leq \int_{|\xi| \leq R}\left(1+R^{2}\right)^{s}\left|\hat{v}_{t}(\xi)\right|^{2} d \xi+\int_{|\xi|>R}\left(1+|\xi|^{2}\right)^{-1}\left(1+|\xi|^{2}\right)^{s+1}\left|\hat{v}_{t}(\xi)\right|^{2} d \xi \\
& \leq C\left(1+R^{2}\right)^{s}\left\|v_{t}\right\|_{2}^{2}+\left(1+R^{2}\right)^{-1}\left\|v_{t}\right\|_{H_{\bullet+1}}^{2},
\end{aligned}
$$

and it follows that $\left\|v_{t}\right\|_{H_{0}} \rightarrow 0$ as $t \rightarrow t_{0}$. The continuity is proved.

We shall now prove (1). Duhamel's formula gives

$$
u(\cdot, t)=e^{i t P} f+\int_{0}^{t} e^{i(t-\tau) P}(-i V u(\cdot, \tau)) d \tau, \quad f \in H_{m} .
$$


For $f \in \mathcal{S}$, we multiply by $\varphi \in \mathcal{A}$ and take norms in $H_{s}, s \geq 0$, getting

$$
\|\varphi u(\cdot, t)\|_{H_{0}} \leq\left\|\varphi e^{i t P} f\right\|_{H_{s}}+\int_{I_{t}}\left\|\varphi e^{i(t-r) P}(V u(\cdot, \tau))\right\|_{H_{\bullet}} d \tau
$$

where $I_{t}=[0, t], t \geq 0$, and $I_{t}=[t, 0], t<0$. Hence,

$$
\begin{aligned}
\left(\int_{-T}^{T}\|\varphi u\|_{H_{\bullet}}^{2} d t\right)^{1 / 2} \leq & \left(\int_{-T}^{T}\left\|\varphi e^{i t P} f\right\|_{H_{\bullet}}^{2} d t\right)^{1 / 2} \\
& +\left(\int_{-T}^{T}\left(\int_{I_{\mathrm{t}}}\left\|\varphi e^{i(t-\tau) P}(V u(\cdot, \tau))\right\|_{H_{\bullet}} d \tau\right)^{2} d t\right)^{1 / 2} .
\end{aligned}
$$

We invoke (5) with $\varrho=s, r=0$ to estimate the first term on the right-hand side.

Using an obvious notation, we have

$\|\varphi u\|_{L^{2}\left(-T, T ; H_{\bullet}\right)} \leq C\|f\|_{H_{\bullet-\alpha}}+\left(\int_{-T}^{T}|t|\left(\int_{I_{t}}\left\|\varphi e^{i(t-\tau) P}(V u(\cdot, \tau))\right\|_{H_{\bullet}}^{2} d \tau\right) d t\right)^{1 / 2}$,

where we have also used the Cauchy-Schwarz inequality in the last term. The square of this term can be majorized by

$$
T \int_{-T}^{T} \int_{-T}^{T}\left\|\varphi e^{i t_{1} P}(V u(\cdot, \tau))\right\|_{H_{\diamond}}^{2} d t_{1} d \tau .
$$

Invoking (5) again, one finds that this is less than

$$
C \int_{-T}^{T}\|V u(\cdot, \tau)\|_{H_{s-\alpha}}^{2} d \tau
$$

(where $C$ depends on $T$ ). Hence,

$$
\|\varphi u\|_{L^{2}\left(-T, T ; H_{s}\right)} \leq C\|f\|_{H_{s-\alpha}}+C\|V u\|_{L^{2}\left(-T, T ; H_{s-\alpha}\right)},
$$

and from (6) and (7) we obtain

$$
\|\varphi u\|_{L^{2}\left(-T, T ; H_{s}\right)} \leq C\|f\|_{H_{s-\alpha}} .
$$

This estimate yields (1) in the case $r=0$. that

We shall now extend this to the case $r>0$. An easy consequence of (9) is

$$
\left\|\varphi D_{x}^{\beta} u\right\|_{L^{2}\left(-T, T ; H_{s}\right)} \leq C\|f\|_{H_{\bullet+|\beta|-\alpha}} .
$$


Indeed, Leibniz' rule shows that

$$
\varphi D^{\beta} u=D^{\beta}(\varphi u)+\sum_{|\gamma| \leq|\beta|-1} \varphi_{\gamma} D^{\gamma} u
$$

for some $\varphi_{\gamma} \in \mathcal{A}$. A simple induction argument gives (10).

It follows from the Schrödinger equation that for $\varphi \in \mathcal{A}$

$$
\varphi \frac{\partial u}{\partial t}=\sum_{|\gamma| \leq m} \varphi_{\gamma} D^{\gamma} u
$$

where $\varphi_{\gamma} \in \mathcal{A}$. Differentiating with respect to $t$ and using the Schrödinger equation again, one obtains more generally

$$
\varphi \frac{\partial^{k} u}{\partial t^{k}}=\sum_{|\gamma| \leq k m} \varphi_{\gamma} D^{\gamma} u, \quad k=0,1,2, \ldots
$$

where $\varphi_{\gamma} \in \mathcal{A}$.

The above equality combined with (10) now gives

$$
\left\|\varphi \frac{\partial^{k} u}{\partial t^{k}}\right\|_{L^{2}\left(-T, T ; H_{\diamond}\right)} \leq C\|f\|_{H_{\bullet+m k-\alpha}}, \quad \varphi \in \mathcal{A}, k=0,1,2, \ldots
$$

It follows that

$$
\|\varphi \psi u\|_{H_{s, k}} \leq C\|f\|_{H_{s+m k-\alpha}}, \quad s \geq 0, k=0,1,2, \ldots
$$

for $\varphi \in \mathcal{A}, \psi \in C_{0}^{\infty}(\mathbf{R})$.

Theorem 1 now follows from the above inequality and interpolation.

\section{The remaining proofs}

Proof of Theorem 2. Here we use the notation

$$
\|S f\|_{L^{2}\left(H_{r}\right)}=\left(\int_{\mathbf{R}^{n}}\|S f(x, \cdot)\|_{H_{r}(\mathbf{R})}^{2} d x\right)^{1 / 2},
$$

so that $L^{2}\left(H_{r}\right)=H_{0, r}$. For $s>\frac{1}{2}$ define $r>\frac{1}{2}$ by $m r-\alpha=s$. Then Theorem 1 implies that

$$
\|S f\|_{L^{2}\left(H_{r}\right)} \leq C\|f\|_{H_{s}} .
$$

Since $r>\frac{1}{2}$ the $L^{\infty}(\mathbf{R})$ norm is majorized by the $H_{r}(\mathbf{R})$ norm. We obtain

$$
\left(\int_{B} u^{*}(x)^{2} d x\right)^{1 / 2} \leq C_{B}\|f\|_{H_{s}}
$$

for every ball $B$, if we choose $\varphi$ and $\psi$ suitably. Theorem 2 is proved.

We omit the proof of Theorem 3, since it is the same as the last part of the proof of Theorem 2 in [5]. 
Proof of Theorem 4. Set

$$
v(x, t)=\int_{\mathbf{R}^{n}} e^{i x \cdot \xi} e^{i t|\xi|^{2}} \hat{f}(\xi) d \xi, \quad x \in \mathbf{R}^{n}, t \in \mathbf{R}, f \in \mathcal{S}\left(\mathbf{R}^{n}\right) .
$$

It is sufficient to prove that if

$$
\|\varphi \psi v\|_{L^{2}\left(\mathbf{R}^{n+1}\right)} \leq C\|f\|_{H_{\bullet}}, \quad f \in \mathcal{S},
$$

for all $\varphi \in C_{0}^{\infty}\left(\mathbf{R}^{n}\right), \psi \in C_{0}^{\infty}(\mathbf{R})$, then $s \geq-\frac{1}{2}$.

Choose $g \in C_{0}^{\infty}(\mathbf{R})$ so that $0 \notin \operatorname{supp} g, g$ is even, and $g\left(\frac{1}{2}\right)=1$. We first assume $n=1$ and define $f$ by $\hat{f}(\xi)=g(\xi / N)$ where $N>1$. Then

$$
\|f\|_{H_{s}}^{2}=\int_{\mathbf{R}}\left(1+|\xi|^{2}\right)^{s}|g(\xi / N)|^{2} d \xi=\int_{\mathbf{R}}\left(1+N^{2}|\eta|^{2}\right)^{s}|g(\eta)|^{2} d \eta N \leq C N^{2 s+1}
$$

so that

$$
\|f\|_{H_{s}} \leq C N^{s+1 / 2} .
$$

The function $e^{i \xi^{2}}$ has Fourier transform $c e^{-i x^{2} / 4}$ for some constant $c \neq 0$, and one finds that

$$
\begin{aligned}
v(x, t) & =c t^{-1 / 2} \int_{\mathbf{R}} e^{-i(x-y)^{2} / 4 t} N \hat{g}(N y) d y \\
& =c t^{-1 / 2} \int_{\mathbf{R}} e^{-i x^{2} / 4 t} e^{2 i x y / 4 t} e^{-i y^{2} / 4 t} N \hat{g}(N y) d y
\end{aligned}
$$

for $t>0$. Hence,

$$
|v(x, t)|=|c| t^{-1 / 2}\left|\int_{\mathbf{R}} e^{i(x / N t) z / 2} e^{-i\left(1 / 4 N^{2} t\right) z^{2}} \hat{g}(z) d z\right|, \quad t>0 .
$$

The function

$$
F(\alpha, \beta)=\int_{\mathbf{R}} e^{i \alpha z / 2} e^{-i \beta z^{2}} \hat{g}(z) d z
$$

is a continuous function of $(\alpha, \beta)$, and $F(1,0)=2 \pi g\left(\frac{1}{2}\right)=2 \pi$. It follows that there exists a $\delta>0$ so that $|F(\alpha, \beta)| \geq 1$ if $1 \leq \alpha \leq 1+\delta$ and $0 \leq \beta \leq \delta$. We conclude that $|v(x, t)| \geq c t^{-1 / 2}$ for $t \geq 1 / 4 N^{2} \delta$ and $N t \leq x \leq N t+\delta N t$. When $1 / N \delta \leq t \leq 2 / N \delta$, one then has $|v(x, t)| \geq c N^{1 / 2}$ for $x$ in an interval of length $\delta N t \geq 1$. Hence, $|v(x, t)| \geq c N^{1 / 2}$ for $(x, t)$ in a set of measure $\geq c / N$.

It follows that

$$
\|\varphi \psi v\|_{L^{2}\left(\mathbf{R}^{n+1}\right)} \geq c N^{1 / 2}\left(\frac{1}{N}\right)^{1 / 2}=c
$$


and (11) and (12) yield $N^{s+1 / 2} \geq c$ for $N>1$, so that $s \geq-\frac{1}{2}$.

When $n \geq 2$, the argument is similar. One chooses $g$ as above and sets

$$
\hat{f}(\xi)=g\left(\xi_{1} / N\right) g\left(\xi_{2} / N\right) \cdots g\left(\xi_{n} / N\right), \quad \xi=\left(\xi_{1}, \ldots, \xi_{n}\right) .
$$

Proof of Theorem 5. Set $u_{0}(x, t)=\left(e^{i t P} f\right)(x)$ and $u_{0}^{*}(x)=\sup _{0<t<1}\left|u_{0}(x, t)\right|$. It was proved in [7] that

$$
\left(\int_{B} u_{0}^{*}(x)^{2} d x\right)^{1 / 2} \leq C_{B}\|f\|_{H_{\gamma}}, \quad f \in \mathcal{S}
$$

where $\gamma=n / 4$.

Since $\left(e^{i t P}(V u(\cdot, \tau))\right)(x)$ is a continuous function of $(x, t, \tau)$ if $f \in \mathcal{S}$, it follows from Duhamel's formula that

$$
u(x, t)=u_{0}(x, t)+\int_{0}^{t}\left(e^{i(t-\tau) P}(-i V u(\cdot, \tau))\right)(x) d \tau .
$$

Hence for $0<t<1$

$$
|u(x, t)| \leq u_{0}^{*}(x)+\int_{0}^{1} \sup _{0<t<1}\left|\left(e^{i t P} e^{-i \tau P}(V u(\cdot, \tau))\right)(x)\right| d \tau .
$$

Using (13) twice we obtain

$$
\begin{aligned}
\left(\int_{B} u^{*}(x)^{2} d x\right)^{1 / 2} \leq & \left(\int_{B} u^{*}(x)^{2} d x\right)^{1 / 2} \\
& +\int_{0}^{1}\left(\int_{B}\left(\sup _{0<t<1}\left|\left(e^{i t P} e^{-i \tau P}(V u(\cdot, \tau))\right)(x)\right|^{2} d x\right)^{1 / 2} d \tau\right. \\
\leq & C\|f\|_{H_{\gamma}}+C \int_{0}^{1}\left\|e^{-i \tau P}(V u(\cdot, \tau))\right\|_{H_{\gamma}} d \tau \\
\leq & C\|f\|_{H_{\gamma}}+C \int_{0}^{1}\|u(\cdot, \tau)\|_{H_{\gamma}} d \tau \leq C\|f\|_{H_{\gamma}},
\end{aligned}
$$

and the proof is complete. 


\section{References}

[1] Constantin, P., and J.C. Saut: Effets régularisants locaux pour des équations dispersives générales. - C. R. Acad. Sci. Paris 304, Série I, 1987, 407-410.

[2] Constantin, P., and J.C. SaUt: Local smoothing properties of dispersive equations. J. Amer. Math. Soc. 1, 1988, 413-439.

[3] Melin, A.: Some mathematical problems in inverse protential scattering. - Séminaire sur les équations aux dérivées partielles 1986-1987, École Polytechnique, no. XX, 1987.

[4] Melin, A.: Intertwining methods in multidimensional scattering theory I. - Preprint 1987:13, Department of Mathematics, University of Lund and Lund Institute of Technology, 1987.

[5] SJögREN, P., and P. SJölin: Convergence properties for the time-dependent Schrödinger equation. - Ann. Acad. Sci. Fenn. Ser. A I Math. 14, 1989, 13-25.

[6] Sjögren, P., and P. SJölin: A maximal function estimate for the time-dependent Schrödinger equation with small potential. - Research Report 1988:11, Department of Mathematics, Chalmers University of Technology and University of Göteborg.

[7] Sü̈lin, P.: Regularity of solutions to the Schrödinger equation. - Duke Math. J. 55, 1987, 699-715.

Peter Sjögren

Chalmers University of Technology

University of Göteborg

Department of Mathematics

S-412 96 Göteborg

Sweden

Received 15 February 1989
Per Sjölin

Uppsala University

Department of Mathematics

Thunbergsvägen 3

S-752 38 Uppsala

Sweden 